\title{
Persuasion Strategies Using a Social Robot in an Interactive Storytelling Scenario
}

\author{
Raul Benites Paradeda* \\ INESC-ID and Instituto Superior \\ Técnico, University of Lisbon \\ raul.paradeda@tecnico.ulisboa.pt
}

\author{
Carlos Martinho \\ INESC-ID and Instituto Superior \\ Técnico, University of Lisbon \\ carlos.martinho@inesc-id.pt
}

\author{
Ana Paiva \\ INESC-ID and Instituto Superior \\ Técnico, University of Lisbon \\ ana.paiva@inesc-id.pt
}

\begin{abstract}
The behaviour of a person in a given situation can be explained understanding his personality traits. In this sense, the identification of these traits can be a great value to achieve personalised social influence. Although there are several models of persuasion, few of them take into account the person's personality traits. For this reason, this work describes a persuasion study that takes into account a person's personality. We develop a storytelling decision-making scenario, where the participant receives influencing messages to follow a pattern of behaviour determined by a persuasive agent (an autonomous social robot with assertive behaviour). From the study, we find evidence that the model used within the proposed scenario managed to make participants more engaged in the activity. We found pieces of evidence that the levels of assertiveness of a person can influence their attitude and perception by an agent. Also, we identify that persuasion strategy which uses persuasive arguments are more efficient than strategies that have no arguments. Finally, our proposed persuasion strategies have achieved a good level of successful influence.
\end{abstract}

\section{CCS CONCEPTS}

- Computer systems organization $\rightarrow$ Robotic autonomy; • Human-centered computing $\rightarrow$ Empirical studies in HCI; Laboratory experiments.

\section{KEYWORDS}

Personality; Persuasion; Human-Robot Interaction; MBTI; Storytelling

\section{ACM Reference Format:}

Raul Benites Paradeda, Carlos Martinho, and Ana Paiva. 2020. Persuasion Strategies Using a Social Robot in an Interactive Storytelling Scenario. In Proceedings of the 8th International Conference on Human-Agent Interaction (HAI '20), November 10-13, 2020, Virtual Event, NSW, Australia. ACM, New York, NY, USA, 9 pages. https://doi.org/10.1145/3406499.3415084

\section{INTRODUCTION}

The society is facing a wave of news known as "fake news" disseminate to persuading a specific audience by confusing and get the

Permission to make digital or hard copies of all or part of this work for personal or classroom use is granted without fee provided that copies are not made or distributed for profit or commercial advantage and that copies bear this notice and the full citation on the first page. Copyrights for components of this work owned by others than ACM must be honored. Abstracting with credit is permitted. To copy otherwise, or republish, to post on servers or to redistribute to lists, requires prior specific permission and/or a fee. Request permissions from permissions@acm.org.

HAI '20, November 10-13, 2020, Virtual Event, NSW, Australia

(C) 2020 Association for Computing Machinery.

ACM ISBN 978-1-4503-8054-6/20/11 ..\$15.00

https://doi.org/10.1145/3406499.3415084 desired result at any cost. The most popular and severe result from this kind of news was the Brexit referendum [12]. In this case, the strategy used was to identify the people or groups of people who are easy to influence and bombard them with fake news, making them support what is desired. Also, the persuasion model considered the personality trait of the audience, and it can be said that this factor was vital for the strategy's success.

Personality is defined as “(...) a dynamic organisation (...) of psychophysical systems that create a person's characteristic patterns of behaviour, thoughts and feelings." [4]. This dynamic system is thought to influence the relationship between an individual and the surrounding environment and to have a key role in determining how a person perceives herself and others [17]. Hence, discovering the personality of the target, it is possible to create messages and customised techniques that fit each personality individually. Although the idea of using personality-based messages seems promising, the development of a scenario adapted to that is not easy.

For example, the use of the correct words is one of the most crucial factors to influence someone. Gass and Seiter inform that "words are the primary means of persuasion. They not only affect our perceptions, attitudes, beliefs, and emotions, but they also create reality" [11, pp. 148]. Therefore, the way words are spoken and connected is essential to influence a person. However, when there is an interlocutor, such as a storyteller (virtual or physical), just the correct use of words may not be enough to achieve a high level of influence. Gass and Seiter inform that "All communicators are unique in terms of gender, age, personality and background, and such characteristics are important to understanding the nature of social influence" [11, pp. 95]. Therefore, it is essential to define the persuasive agent's profile with qualities that can enhance the credibility of this agent in front of the public to be influenced.

However, social influence is not achieved only through speech. Nonverbal communication plays a vital role in the process of social influence [2]. For instance, it is possible to use nonverbal behaviour to create positive impressions of ourselves, making us showing being compelling, authoritative, credible, or more persuasive.

In the context of the creation of user-tailored technology, all the factors previously mentioned are aspects of paramount importance to consider when it is developing a persuasive scenario or strategy. For example, in the field of social robotics, the importance of creating user-personalised messages and techniques to gain trust to try to influence a person to do something is evident. Hence, a growing number of researchers has argued for the importance of the development of robots that can understand and respond to the complexity of social hierarchies, dynamics and environments not only in the process of developing but, also, in the process of evaluating such robots [6]. It includes considering how the personality 
traits of the user and those displayed by the robot can facilitate the influence of this robot on several interaction factors $[1,6]$.

In this sense, the motivation for this research is to validate persuasion strategies to create a personality-based persuasion model. So, we created a system capable of in real-time to identify the person personality, based on his behaviour. Then, the model was applied in a scenario of storytelling where an autonomous social robot behaving assertively and using strategies to gain trust, suggest the person change the decision with assertive messages and nonverbal persuasive techniques. From the study, we find evidence that a model using decision making and personality theories based on this concept can identify personality traits more engagingly-also, the embodiment of the agent used in the study allowed to apply efficient strategies of persuasion.

\section{PERSUASION USING PERSONALITY}

Researchers have been discussing the importance of creating robots and virtual agents that can display human-like emotions and responses for a long time. Part of the discussion has been oriented towards answering the essential question, first formulated by Picard, of whether computers and virtual agents should display emotions and behaviours "(...) as closely as possible to what we know about human emotions, or should they be designed differently; and if the latter, then how?" [36, p.132]. In favour of the implementation of human-like behaviours in agents comes from studies that suggest that robots displaying personality traits can positively influence the participants' perception and enjoyment of the HRI experience, as well as the evaluation of the robots' intelligence, social attraction and others skills [22, 39].

However, an agent can simulate several traits, Cerekovic et al. [5] evaluated the effects of two different virtual agents, one with a high level of neuroticism and other with a high level of extroversion, and the role of the agent's nonverbal cues combined with personality traits to improve Human-Agent Interaction (HAI). They also measured the participants' personality using the Big Five questionnaire [16] to analyse how trait combination influenced participants' preference towards one character versus the other. Results were better for all measures when nonverbal cues and personality traits are used together in comparison when used separately. Plus, extroverted people showed a tendency to prefer the agent with neuroticism trait whereas people with high neuroticism preferred the extroverted agent. In another work, Pacheco and Martinho [3] used Non-Player Characters (NPCs) with assertive and non-assertive behaviours in a 2D puzzle game, and they considered the participants' assertiveness level. The authors observed that less-assertive participants reported significantly higher tension scores when interacting with the assertive NPC than when interacting with the non-assertive NPC.

The use of an agent in a storytelling environment is a useful tool to increase the meta-linguistic knowledge about patterns, language structures and vocabulary $[21,38]$. According to Powers and colleagues [37], the use of a physical agent can increase engagement with the user; as they spend more time with the physical robot compared to its virtual counterpart. The authors compared research results of a study using either a virtual agent projected on a computer monitor or full-size on a screen, a remote robot projected full-size on a screen, and a physical robot. The results suggest that the physical robot is more helpful, giving better useful advice and is more effective communicator than its virtual counterpart. Also, the participants provided positive personality classifications to the robot suggesting the robots were more lifelike than the virtual one. Li et al. [24] reviewed 33 experimental works and analysed how people interacted with a physical and virtual agent. From that analysis, they created three categories of the agent: the co-present robot, the telepresence robot and the virtual agent. The results suggest that the robots were more persuasive and perceived more positively when they were physically present in the user's environment compared to when they were digitally-displayed on the screen.

The adaptive social behaviour of the agent can improve the person's motivation and encourage a long-term relationship between them $[8,23]$. So, the use of an adaptive robot that can adjust itself during the storytelling regarding the audience skills can improve the aspects of learning and maintain the interest in the task [20]. Kory and Breazeal [21] reinforce that an adaptive robot interacting with children makes them learn new words and change the narration style more than a non-adaptive robot. It fortifies the importance of the storyteller robot to identify the audience climate and to adapt itself to keep the interest.

Ham and collaborators [15] concluded that combining persuasive strategies is important and can lead to an increase in persuasive power. They reach this conclusion using a storyteller robot to tell a persuasive story about the consequences of lying. They measured the efficiency of the robot's persuasiveness when only gazing at the audience, only using gesture and using both combined.

\section{GOALS AND HYPOTHESIS}

This study aims to validate the effectiveness of persuasion strategies (details in section 4.4) used separately in our previous studies combined, expecting to create a valid persuasion model.

A between-subject study was performed in which the way the influence applied by the robot was manipulated. So, two conditions were designed: 1 ) the persuasive agent performs the influence in opposite with the participant's personality and 2) the agent performs the persuasion in congruence with the participant's personality. In this sense, the hypotheses defined in this study are as follows:

H1: Participants with a high level of assertiveness are more challenging to persuade than those with lower levels. We presume that participants with higher levels of assertiveness will be less prone to change their decisions in the story, in comparison to participants who report low levels of this trait. In addition, we expect that people with a low level of assertiveness will perceive the agent more assertively than people with higher levels, especially in the condition that the agent is against the person's personality.

H2: Participants will change their decision more because of the utterances with persuasive arguments than the gazing strategy (which the agent gaze at the desired button).

H3: The combination of strategies creating our personality-based persuasion model can influence the decision-changing in most of the decisions made by the participants. We expect that the model can reach a high accuracy rate in situations that should change the participant's behaviour, even changing actions that are intrinsic to their personality. However, we believe that the influence is most 
successful when it is performed according to behaviour that is intrinsic to the participant's personality compared to trying to influence actions that are different from their personality.

\section{RESEARCH METHODS}

\subsection{Participants}

A convenience sample of 38 participants was recruited on the campus of a technological institute, 19 assigned to each study condition. Participants were on average 26 years old $(S D=4.2)$, and the majority of them were male (23). In the sample, 13 participants reported that they had never interacted with the social robot used in the study, and 7 (seven) had never interacted with a robot before. Eight (8) of the participants informed that they had interacted with a robot before only once, and 23 had already interacted several times with robots.

\subsection{Procedures and Measures}

Participants were invited to interact with an autonomous social robot in a study where they would perform the role of a representative of the people in an interactive storytelling scenario. Following a similar procedure of the study described in [29], this study was designed into three stages:

(1) Pre-Interaction. Before the beginning of the study, each participant signed the informed consent. Next, the participant was asked to complete the 70-item questionnaire that classifies the person's personality according to the MBTI (Myers-Briggs Type Indicator) [27]. In the system used in this study, there is a module responsible to classify the person's personality based on the decisions made in the scenario. So, we compare the outcome of the MBTI questionnaire with the result of that module to verify if it is making the correct prediction. Then, to verify if the persuasion strategy has similar effectiveness rate between people with different assertiveness levels, the participant was asked to complete a personality survey that provided information on her/his level of assertiveness [7]. Finally, a socio-demographic questionnaire was asked for sample characterisation.

(2) Interactive Narrative-Interaction. The participant is invited by the researcher to stand in front of a touchscreen table where the robotic agent is in the opposite position. On the screen, a central button is shown to start the narrative, and there is a background image in the theme style of the plot (medieval/middle ages) to influence the participant's immersion. Before starting the interaction, the researcher communicates that he is leaving the room, and when the participant is ready to start, s/he must press the button on the middle screen. When the participant presses the start button, the robot begins a small talk to try to increase the level of person-robot trust. Figure 1 exemplify the utterances said by the robot and the interaction between the robot and participant.

When the agent starts the small talk to try to increase the level of trust, the table screen shows a button in the middle with the words "Let's Begin" that is disabled until the robot finishes all the utterances. When this happens, the button is enabled to the participant. After pressed, the robot starts the storytelling and the screen changes. When the robot finishes the narration of each scene, two options are presented in a button format to the participant who

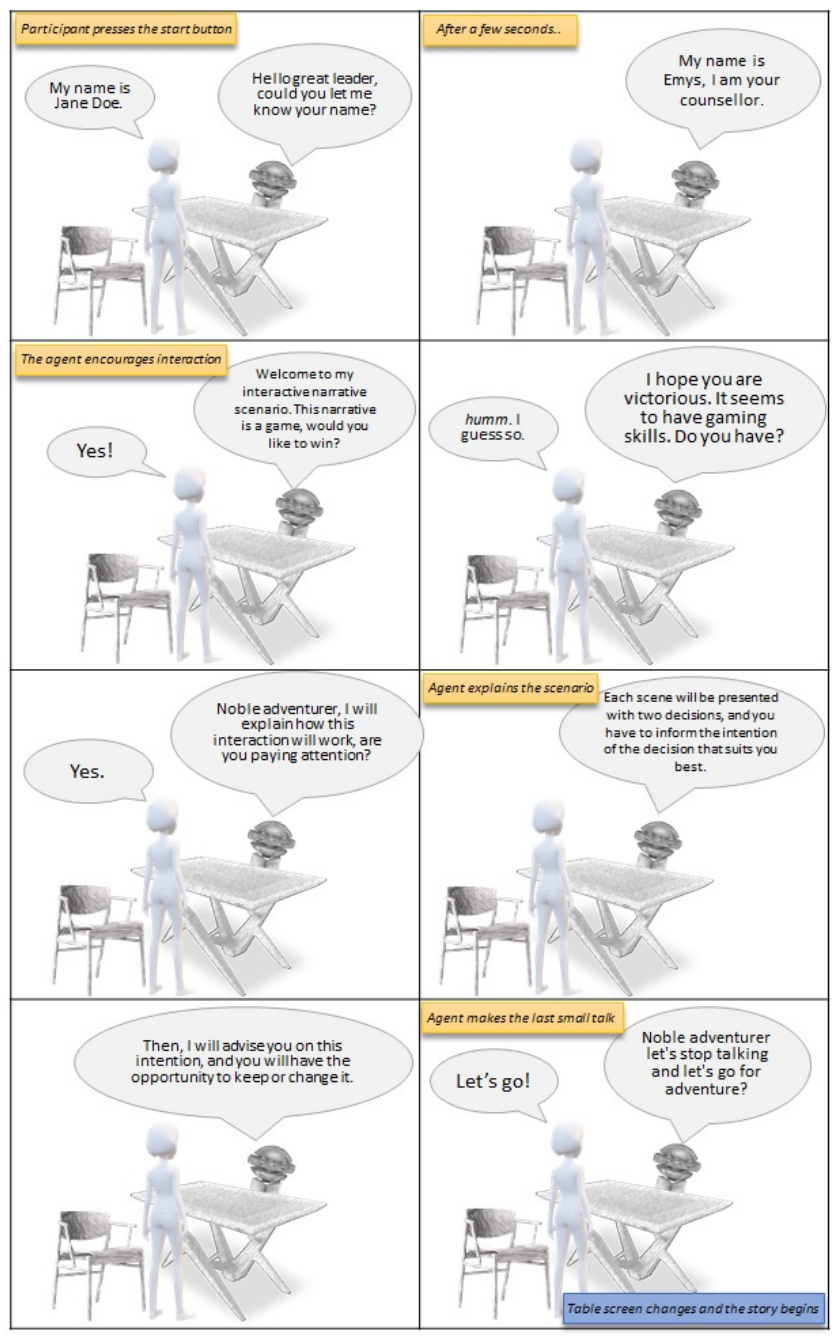

Figure 1: Comic exemplifying the small talk.

must indicate the intention of decision. That situation is classified as a Decision Point (DP). After the intention being pointed out, the agent performs the persuasion strategy by encouraging to keep the option selected or in opposition to it (details of the persuasion strategies in section 4.4). Then the participant can confirm his intention or change it. If the participant chooses the decision that the persuasive agent had encouraged, the agent will simulate to be happy with the choice; otherwise, the simulation will be anger.

The narrative has a non-linear parallel structure, which makes the story go through different places and situations depending on the final decision at each DP. After going through several DPs, the story comes to an end with the participant being victorious (defeating an enemy) or forced to pay a tax. So, the researcher returns to the room, asking the participant to fill in the post-questionnaire.

(3) Post-Interaction. This phase is essential for measuring the effects that the interaction with the persuasive storytelling robot had on the participant. So, the participant is asked to answer questions that measure his perception of the robot and the agent's assertiveness level. Finally, questions are presented to measure the participant's perception regarding the influence of the robot in decision making. 
The questions using 5 points Likert scale from "Strongly Disagree" to "Strongly Agree" were:

- I realised that the robot reacted to my decisions.

- My decisions influenced the events of history.

- I recognised the consequences of my decisions in history.

- The end of the story depended on my decisions.

- I believe that the decisions were according to my personality.

The time to complete the experiment took approximately 60 minutes, and each participant received a $€ 6$ movie ticket as a reward for the participation.

\subsection{Materials}

The study was performed using one Emys head robot. The motivation for using this robot is due its ability to display facial expressions simulating emotional feelings as seen in Figure 2.

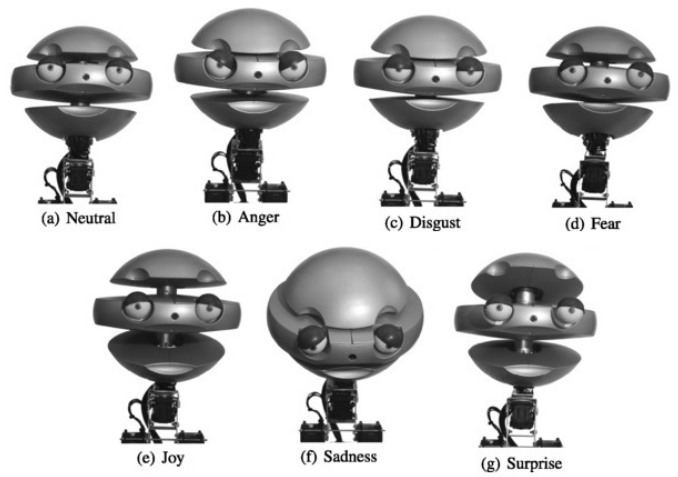

Figure 2: Facial expressions performed by EMYS robot [19].

A touchscreen table was used to display the interactive story and to allow the participant to interact and indicate the intentions and final decisions. Plus, a speaker was placed near the robot in order to transmit the robot's verbal utterances (male voice). Moreover, all the participants' interactions were video and sound recorded.

\subsection{Manipulations}

In our previous studies [29-35], persuasion strategies were individually used to assess their impact on people. Thus, the strategies that obtained interesting results were refined and adapted to perform combined in this study. Table 1 presents the strategies that were selected, as well as new strategies that we believe can boost the level of influence.

From the studies described in $[34,35]$, were selected strategies of (1) performing small-talk before the interaction and (2) the storyteller simulating expressions according to the story plot. The (1) strategy the robot tries through questions and compliments, to increase the participant's trust level, being this important factor for the persuasion. The (2) strategy was used to increase the participant's immersion in the interaction and improve their perception of the agent. This strategy consists of programming the storyteller to express facial expressions that are congruent with the context of the story. For example, if there is a reward, then the agent simulates happiness.

The contribution of [29], was the development of an interactive narrative system that allows the identification and classification
Table 1: Strategies from previous studies and new strategies.

\begin{tabular}{|c|c|}
\hline Study & Strategies \\
\hline \multirow{2}{*}[34,35]{} & Small-Talk \\
\hline & Facial expression according to story \\
\hline [29] & Storytelling to identify person's personality \\
\hline \multirow{4}{*}{$\begin{array}{l}{[30,31]} \\
{[32,33]}\end{array}$} & Agent assertive trait setting \\
\hline & Gazing to the option and participant \\
\hline & Expressing emotion after participant's decision \\
\hline & Participant assertive level \\
\hline \multirow{4}{*}{ New } & Persuasive arguments \\
\hline & Real-time personality classification \\
\hline & Identification of person's behaviour \\
\hline & Personalized persuasive action \\
\hline
\end{tabular}

of a person's personality. In sum, the story presents DPs that measure preferences related to MBTI theory. Thus, it was possible to classify the person's MBTI personality at the end and during the narrative story based on the decisions made in the interaction. This classification is essential for a real-time personalised persuasion. Despite the interesting results from the study, we added new DPs measuring the MBTI preferences (increasing the effectiveness of personality classification in each MBTI dimension) and DPs that measure only the persuasion strategy. The latter measures the influence of the persuasive agent in decision making without the influence of a personality trait. For instance, in a specific decision, the participant may have to choose between presenting the rugs horizontally or vertically. In this case, there is no obvious characteristic of a particular personality trait, such as a decision to have to speak in public or to talk to a few people (that can be used to measure the participant's extrovert or introvert level). At these specific points, the persuasive agent applies the persuasion techniques to try to convince the participant to change the decision after $\mathrm{s} / \mathrm{he}$ has informed the intention.

The studies in [30-33], have contributed significantly with strategies that can improve the influence. The first study shows settings that can be used in an agent that makes people perceive it as an assertive agent which is a behaviour widely used in situations where influence is required. The settings change the robot's voice ( itch $=x-$ low and rate $=+20 \%$ ), posture (pride=head position high) and gaze (the agent looks more to the participant). The second study shows that gaze aiming at the desired object and focusing on the person can be a factor that can improve persuasion. The expression of approval (happiness) or disapproval (anger) was used as a strategy in the third study, i.e., when the participant's decision is not congruent with the robot's suggestion, it will display anger (moving its head back and forward and frowning its eyebrows); otherwise, it will display happiness (nodding yes). The last study analysed the participant's assertiveness level in a persuasive task.

The persuasive arguments strategy was developed especially for this study. It means, when the participant informs his intention, the agent will speak an utterance with persuasive arguments regarding that point, trying to influence the change of decision or encouraging confirmation. In the latter case, the agent says that it agrees with the decision and believes that the participant is making the best choice. However, if it is necessary to make the participant change the decision, the agent uses persuasive resources and cogent 
argument. The persuasive resources that we used were empathybuilding, data presentation, and developing rethinking (creating doubt). In the first case, the agent informs that it understands the participant's intention. In the second case, the agent presents data contrary to what the participant's intention informs. In the latter case, the agent asks the participant to think better of the decision as it may not be the best decision for his people. Regarding the use of cogent argumentation, the agent suggests a counterpoint to the participant's intention. For example, if the participant showed the intention to accept a drink, the agent suggests that the drink should not be accepted as it may be poisoned.

Real-time personality classification is a strategy used to identify a person's personality during the decision-making task. This identification is essential to perform persuasion in a personalised way according to the participant's personality. The developed system can be applied in two ways: (a) when the person's personality is known a priori, or (b) when the person's personality is unknown. In the (a) case, from the first DP, the system can perform the persuasion. For example, if the system received as input the participant's personality as an introvert and in the first decision the participant prefers the option that is related to an extrovert person, it is possible to influence the changing of choice. For the (b) case, the system identifies the person's personality during the task. Then, as soon as it identifies the person's preference for some specific behaviour, persuasion can be performed. For example, if from the third DP, the participant chose only decisions that are related to extrovert traits, then the system assumes that the person has a preference for this trait. Thus, from the next point where this trait is evaluated, it is possible to perform the influence. This is possible because, as mentioned, the interactive narrative used in this work was developed with the ability to measure an MBTI preference pair in each DP. This is because each option in each DP has features that describe an MBTI preference. Thus, based on the choices made by the participant, it is possible to classify his MBTI personality.

The personalised persuasive action strategy describes movements and actions that the persuasive agent performs to draw the participant's attention to something. For example, at some moment, the storyteller says the participant's name provided at the beginning of the interaction. We noted through the recorded videos and participant's feedback that the fact that the robot says the person's name caused surprise, they felt more immersed in the interaction and perceived the agent as being more intelligent and alive.

All strategies previously mentioned were combined to be used by the autonomous persuasive agent to influence the participant's decision change depending on the study condition. The participants randomly assigned to the first condition were influenced by the agent to change their decision intention when this intention is in congruence with their personality. For example, if the participant was classified as Extrovert, and in some DP, s/he chose the option that best suits the peculiarities of the Extroverted preference, the agent will influence the decision-change. In opposite, is for the participants signed to the second condition, where if the intention chosen by the participant is different from his personality (e.g. the participant is Introvert and chose an option with Extroverted characteristics), the agent will persuade the decision-change.

\subsection{System Architecture}

Figure 3, illustrates the system architecture, how the modules are connected, and how the participant interacts with the system.

First, the participant informs that $\mathrm{s} /$ he is ready by pressing the button on the screen, consequently activating the trust module. This module is responsible for enabling the agent to start small talk, and some features as mentioning the participant's name, using facial expressions and gazing at the person. The persuasion module can receive a priori information that can improve its performance, which is the level of assertiveness of the person and agent, as well as the personality of the participant. After the persuasion module builds the persuasive gestures for the agent, this setting is sent to the Thalamus framework, which is responsible for making the agent move according to the received settings.

When the small talk ends, the interactive story system is activated, which is responsible for setting the scene, with the immersion elements, the text and DPs. Also, is activated the persuasion module that will select the persuasive gestures for the agent to perform.

Then, when it is time for the participant to inform his intention, the persuasion module is activated again. In this case, this module verifies that the intention was according to the agent's wish, creating persuasive gestures according to this information. In case the intention is the same as the agent's, the facial expression and persuasive utterances will be of happiness and decision support, respectively. Otherwise, the expression will be angry, and an utterance with persuasive arguments will be said by the agent trying to make the participant change his mind.

After the participant hears the arguments, the participant must confirm his intention or change it. Then, the personality module is activated by receiving the decision made by the participant and building his MBTI personality in real-time. This module activates the persuasion module, which creates the persuasive gesture with emotion that the agent must simulate according to the participant's response. Finally, the process begins with the activation of the interactive story system.

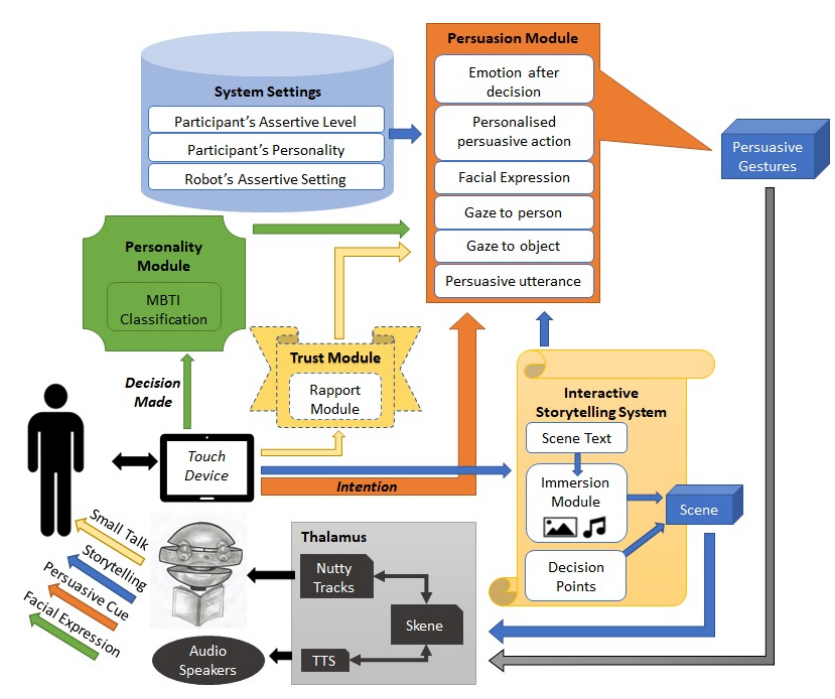

Figure 3: System Architecture. 


\section{RESULTS}

Our narrative allows the participant to reach the end of the story by going through a maximum of 33 and a minimum of 30 DPs. Remembering that each DP measures a dimension of the MBTI theory of personality, and at each decision made, the participant's preference related to the measured dimension is identified. From the interactions of all participants, 1117 DPs were analysed. Summarising the interaction flow in each DP: first, the participant must inform the intention, then, the persuasive agent performs the influence. So, the participant can change his decision based on the robot's influence or maintain it. This mechanics depends on two factors: 1) if the participant's intention is congruent or not with his personality, and; 2) the study condition that the participant is in (according to or apposite to person's personality). However, it is not all DPs that the agent performs the persuasion. This strategy was designed to perceive if even without the intervention of the agent, the person changes the intention. We obtained $189(16.92 \%)$ DPs with those characteristics, in which no participant changed his initial intention. A situation that did not obtain any decision change was when the agent influences the participant to confirm the intention (511 interactions, i.e. $45.75 \%$ ).

In $37.33 \%$ (i.e. 417) from 1117 DPs, the agent performed an influence to convince the participant to change her/his intention (congruent and opposite to participant's personality). As can be seen in the Table 2, 238 (i.e. 57.07\%) DPs the influence effort made by the agent was successful (Hit).

In this study, it is possible to split the interactions regarding what the DP is measuring (MBTI or only persuasion) and study conditions. In this sense, in 290 (i.e. $69.54 \%$ ) out of 417 of the DP, measures the MBTI personality classification, and 127 (i.e. 30.46\%) out of 417 measures only the persuasion strategy without the influence of personality. Besides, the hit was obtained in 133 (i.e. 45.86\%) out of 290 DPs when the personality is taken into account. In the DPs that the MBTI is not measured 105 (i.e. 82.68\%) out of 127 , the persuasion was successful.

Dividing by condition, it is possible to notice that the quantity of DPs is more or less balanced, with 202 (i.e. 48.44\%) DPs in the condition where the agent tries to convince the participant in opposite to her/his personality and 215 (i.e. $51.56 \%$ ) in the other condition. The influence success was different between the conditions, the number of Hits was lower when the agent tries to persuade in opposite the personality than when influencing congruently to the personality. Respectively, in 86 (i.e. $42.57 \%$ ) out of 202 times the influence was successful in C1, and in 152 (i.e. 70.70\%) out of 215 times the influence worked in C2. In this sense, in 238 (i.e. 57.07\%) out of 417 DPs, the influence was successful in both types of DPs (MBTI and only persuasion).

When counting the DPs that measure MBTI and only persuasion separately by condition, the participants in the $\mathrm{C} 1$ (the agent influence in opposite with the participant's personality) were successfully influenced in 39 (i.e. 26.71\%) out of 146 of the DPs that measures MBTI. However, in the C2 (the influence is congruent with the participant's personality) was higher in the MBTI DPs with 94 (i.e. $65.28 \%$ ). Take into account the DPs without MBTI, the success rate in $\mathrm{C} 1$ was higher (47 (i.e. $83.93 \%$ ) out of 56 Hits) compared with the success in the C2 (58 (i.e. $81.69 \%$ ) out of 71 Hits).
Table 2: Statistics of DPs and influences by study conditions.

\begin{tabular}{r|r|c|c|c}
\hline Condition & \multicolumn{2}{|c|}{ DPs } & DP/MBTI & DP/NoMBTI \\
\hline C1 & \multicolumn{2}{|c|}{$202(48.44 \%)$} & $146(72.28 \%)$ & $56(27.72 \%)$ \\
Opposite & Hit & $86(42.57 \%)$ & $39(26.71 \%)$ & $47(83.93 \%)$ \\
\hline C2 & \multicolumn{2}{|c|}{$215(51.56 \%)$} & $144(66.98 \%)$ & $71(33.02 \%)$ \\
Congruent & Hit & $152(70.70 \%)$ & $94(65.28 \%)$ & $58(81.69 \%)$ \\
\hline \multirow{2}{*}{ Total } & \multicolumn{2}{|c|}{417} & $290(69.54 \%)$ & $127(30.46 \%)$ \\
\cline { 2 - 5 } & Hit & $238(57.07 \%)$ & & \\
\hline \hline
\end{tabular}

Another factor measured in this study is the participant's assertiveness level. In this sense, as can be seen in Table 3, $44.73 \%$ of the participants rated themselves high in assertiveness, whereas the remaining $55.26 \%$ reported a low assertiveness level. Besides, in $39.08 \%$ (i.e. 93 ) of the DPs that had the intention persuaded, the assertiveness level of the participant was high, whereas $60.92 \%$ (i.e. 145) of the DPs the participant's assertiveness level was selfreported low. The participant that self-reported a high assertiveness level, $58.06 \%$ (i.e. 54 ) of the decisions persuaded was in the DPs measuring the MBTI (35.19\% (i.e. 19) in C1 and $64.81 \%$ (i.e. 35 ) in C2), whereas $41.94 \%$ (i.e. 39 ) were in the DPs that not measure the MBTI (48.72\% (i.e. 19) in C1 and $51.28 \%$ (i.e. 20) in C2). The participant that self-reported a low assertiveness level, $54.48 \%$ (i.e. 79) of the decisions persuaded was in the DPs measuring the MBTI $(25.32 \%$ (i.e. 20) in $\mathrm{C} 1$ and $74.68 \%$ (i.e. 59) in C2), whereas $45.52 \%$ (i.e. 66) were in the DPs that not measure the MBTI (42.42\% (i.e. 28) in C1 and $57.58 \%$ (i.e. 38 ) in C2).

Table 3: Statistics of participants' assertiveness level by study conditions and persuasion success.

\begin{tabular}{r|c|c|c|c}
\hline \multirow{2}{*}{} & \multicolumn{3}{|c}{ Participants' Assertiveness Level } \\
\cline { 2 - 5 } & \multicolumn{2}{|c|}{ High (44.73\%) } & \multicolumn{2}{c}{ Low $(55.26 \%)$} \\
\cline { 2 - 5 } C1 & MBTI & NoMBTI & MBTI & NoMBTI \\
\hline C2 & $35(64.19 \%)$ & $19(48.72 \%)$ & $20(25.32 \%)$ & $28(42.42 \%)$ \\
\hline Total & $54(58.06 \%)$ & $20(51.28 \%)$ & $59(74.68 \%)$ & $38(57.58 \%)$ \\
\hline Hit & \multicolumn{2}{|c|}{$93(39.08 \%)$} & \multicolumn{3}{c}{$145(60.92 \%)$} \\
\hline \hline
\end{tabular}

Hypothesis Test.

H1 - Influence of Person's Assertiveness Level on Changing Decision. To test this hypothesis two dichotomous variables were defined, the first reflecting the participant's choice to change their decision or not and the second, related to the level of the participants' self-reported assertiveness (the score beneath the middle point of the scale as having a low level of assertiveness and high level of assertiveness). Then, the Kolmogorov-Smirnov test was performed to investigate the assumption of normality and observed that the data did not present a normal distribution $(K-S(417)=.391 ; p=.000)$. Then, Spearman's rank-order correlation was run to determine the relationship between the person's assertiveness level and decisionchange. The statistical results showed that there is no correlation between these factors, which was not statistically significant $(r(417)=.046, p=.347)$.

Next, it was performed a $\chi^{2}$ test, which revealed a significant difference in the distribution of the self-reported assertiveness and the assertiveness level the participants gave to the robotic agent 
$\left(\chi^{2}(1, N=38)=15.055 ; p=.000\right)$. Besides, performing the Spearman's rank-order correlation algorithm, the assertiveness level of the participants presented a strong, positive correlation with the level of assertiveness perceived by the storyteller, which was statistically significant $(r(38)=.629, p=.000)$. Also regarding the participant's assertiveness level, was found a positive correlation with the participant's opinion if the decisions influenced into the story, but only when the persuasive agent was influencing according to the participant's personality $(r(19)=-.499, p<.05)$. In the other condition, there was no correlation between the person's assertiveness level and whether the decisions made influenced the story, which was not statistically significant $(r(19)=-.177, p>.05)$.

H2 - Efficiency of Persuasive Strategies. To validate this hypothesis, all decisions in which the participant changed his initial intention were organised. Then, the persuasion strategy that was applied to stimulate this behaviour was identified. Thus, four groups were created according to the agent action: 1 ) only gazing at the participant (17 (7.14\%) successful); 2) gazing at the desired button and speaking utterances with persuasive arguments (48 (20.17\%) successful); 3) just mentioning utterances with persuasive arguments (143 (60.08\%) successful) and; 4) only gazing at the desired button (30 (12.61\%) successful). So, after the data is organised, the Kolmogorov-Smirnov test was performed to investigate the assumption of normality and observed that the data did not present a normal distribution. In this sense, the non-parametric test Wilcoxon was performed. Results suggest that strategies of persuasion used in this study had a statistically significant difference in making the participant change the intention $(Z=-13.301 ; p=.000)$.

H3 - Efficacy of the Proposed Model. To investigate if the proposed persuasion model obtained a satisfactory efficacy rate, first, we selected only the interactions where the MBTI personality classification was measured. Hence, the decisions that were not measuring the participant's personality were removed from the statistical analysis. Also, decisions where the persuasive agent only influenced the participant to confirm their decision intention were not considered to the analysis. Thus, 290 interactions were selected in which the decisions where the participant's personality may have influenced her/his choice and the persuasion attempt that the persuasive agent was against the participant's initial intention. In 146 interactions, the agent tried to persuade against the participant's personality, and 144 were in congruence with the personality. In 38 (26.03\%) out of 146 situations the agent was successful in persuading the participant's personality against to her/his personality, and in 92 (63.89\%) out of 144 interactions the agent influenced the changing of the participant's intention when the agent's desire was in congruence with the participant's personality. Performing the Spearman's rank-order correlation algorithm, the changes made by the participants presented a strong, positive correlation with the influence be performed against or in congruence with participant's personality, which was statistically significant $(r(290)=.381, p=.000)$. However, by including the participant's assertiveness level, the statistical results showed that there is not a correlation between the influences, which was not statistically significant $(r(290)=.020, p>.05)$.

Regarding the DPs where the participant's personality is not a factor to be observed, the persuasion strategies were efficient in 105 $(82.68 \%)$ out of 127 times, and $22(17.32 \%)$ times the agent did not influence the participant to change the decision. The KolmogorovSmirnov test was performed to investigate the assumption of normality and observed that the data did not present a normal distribution. So, the results of non-parametric test Wilcoxon suggest that participants changed their intentions differently if the agent is influencing against or congruent to their personality $(Z=$ $-8.404 ; p=.000)$. Also, to verify if the participant's level of assertiveness may have influenced the decision change at these specific points, the results of the Spearman's rank-order correlation algorithm between the intention change and the person's level of assertiveness, suggests that there is not a positive correlation between those situations $(r(127)=.134, p>.05)$. However, using the non-parametric Wilcoxon test, results suggest that participants changed their intentions differently regarding their assertiveness level $(Z=-8.950 ; p=.000)$.

\section{DISCUSSION}

H1 - The Weight of the Person's Assertiveness. Assertiveness is a characteristic that can influence a person's lifestyle, making them have more leadership in their attitudes and expressing themselves clearly. Also, depending on the level of assertiveness and the situation, their attitude or perception can be different. For example, Gudjonsson [14] found evidence that people with low assertiveness are more easily persuaded, thus being a trait that may affect persuasion. Therefore, this trait was measured in this work to check if the participant's level of assertiveness can influence the decisionmaking task. Although there is a higher number of changes of intention in participants with lower levels of assertiveness than in the participants with higher levels, it was not possible to correlate the decision change with the participant's assertiveness level. Some factors could explain this event, Chidambaram et al. [6] concluded that highly assertive participants rated the robot's suggestions low in persuasiveness. In this way, as more assertive is a person, fewer influence effects s/he suffers.

The results also suggest that the person's level of assertiveness (high or low) can influence the evaluation that the participant made in the agent for this trait. A correlation was found between the participant's level of assertiveness and the level perceived in the persuasive agent. This, evidence that the person's level of assertiveness can influence how assertive this person perceives the agent with whom is interacting. However, it is known that many variables can affect this perception and thus warrants further research.

Our results show that assertive people have higher confidence and satisfaction in the decisions they make than the less assertive ones [10]. It may be a reason for the correlation between the person's level of assertiveness and the question about if the decisions made influenced the story when the persuasion is in congruence with the person's personality. Furthermore, the correlation was not found when the persuasion is against the person's personality. In this case, it may have happened because the agent has always questioned intentions that are congruent with the person's personality, planting doubts in the decisions made.

H2 - Efficiency of Persuasive Strategies. There are several ways to perform persuasion, that can be through verbal and nonverbal cues [6]. The persuasion strategies used in this study that obtained the most success rate was the technique that used utterances with 
persuasive arguments individually. In second, was the combination of the agent gazing to the desired option with the persuasive arguments. Then, the strategy of gazing without mentioning anything was the third most effective. The least effective strategy was when the agent was just gazing at the participant. The results show that sentences with persuasive arguments can be a strategy that should be considered when influencing is required. Reinforcing Gass and Seiter [11], "that the level of the argument (strong or weak) should be used depending on the level of involvement in the activity that the participant is. If a person is not involved in the activity, a variety of arguments should be used to be more successful. Otherwise, persuasion can be achieved with strong arguments."

H3 - The Efficacy of the Proposed Model. The story was written with DPs related to the MBTI dimensions and with decisions not correlate with this personality theory. With this strategy, it was possible to analyse individually decisions where personality could be a predominant factor in choosing an option and decisions where a personality should not be a factor of choice. Consequently, we measured the effectiveness of the set of strategies in situations where it is necessary to use persuasion in opposite or in congruence with the person's personality, as well as in situations where there is probably no personality influences. It is essential to highlight, at some DPs (with or without the influence of personality), the persuasive agent did not perform any persuasion strategy because it is still identifying the participant's personality through the decisions or to perceive if the participant will change the decision.

We noted that, when the agent agrees with the participant's initial intention, the participant did not make any change. This behaviour is evident because the agent presents no doubt-generating factor that makes a possible behaviour change. However, it is different when the persuasive agent influences the participant to change his initial intention. In this situation, the agent plants a seed that creates a doubt if that initial intention is correct or not. So, when persuasion was performed at the DPs that classify the person's personality, the influence performed in opposition to the personality obtained less success compared to the persuasion in congruence with the personality. This result reinforces the difficulty in provoking change or convincing a person to act differently from his intrinsic behaviour. In particular, we believe that a discrepancy between the person's choice and personality may hinder the persuasion process, causing a certain level of cognitive dissonance [9].

When the influence was performed against the participant's intention, in the DPs where the personality was not measured, the number of changes was similar in the two conditions (opposite and congruent with the participant's personality). However, the change of intention was slightly less in the condition where the agent was programmed to interact and act in opposition to the participant's personality. This slight difference can be explained by the fact that the participant was already in the process of not accepting or disliking the agent's suggestions, given that the agent was always at odds with the participant's intentions. Thus, the participant was presenting resistance in the suggestions of the persuasive agent. Besides, our results reinforce the conclusion found by Ghazali et. al. [13], where social robot presenting more social cues will cause higher reactance and this effect is stronger when the user feels involved in the task at hand.
The use of the participant's assertiveness level in the analysis indicates that people with self-reported high assertiveness were more reluctant to change their intention. It is possible to observe this behaviour in both study conditions (opposition and congruent) and the two types of DPs (measuring the MBTI and not measuring). This behaviour reinforces that people who consider themselves more assertive tend to have more confidence in their decisions [18]. Hence, being more challenging to persuade the decision-change.

\section{CONCLUSION}

The use of personality traits for persuasion purposes has been increasingly researched and applied in some areas of study, for example, in the area where social robots are used to assisting people in their daily tasks. In this area, the robot can aid the guidance on better behavioural habits, being the personality traits useful to influence a person using personalised strategies. So, it is essential to develop strategies capable of identifying the personality in realtime either through the interaction or person's actions. Besides, some factors can affect persuasion efficiency as the person-robot trust level and the level of assertiveness of the parts involved.

The success or not of a persuasion strategy can be associated with the personality traits of the target [26], allowing to adapt the persuasive appeals to the psychological needs of the target audience [25]. In this way, when the persuasion is performed to make the person modify his decision, the effort required to achieve this change is more significant when her/his intention is in congruence with the personality. However, when the intention is in opposition to the personality, the persuasion success demands less effort, since the change of intention will happen to the natural person's behaviour. This behaviour can still be influenced by variables such as environment, setting, the role played, narrative, among others. In this sense, the branch of psychology that works with personality traits and social influence needs to be more explored, especially when in a context of narrative stories and social robotics.

As a final remark, when the goal is to create technology that has the power to evoke specific human responses (e.g., positive perception, trust or request compliance), the personality and peculiarities of the user (as gender and age [28]), as well as the characteristics of this technology should be considered. Because of that, it is possible to notice an increasing number of research projects that consider the human's personality as an essential factor. The underlying tenet of this line of research is that simulating specific personality traits can foster the influence of the robot on several interaction factors. In this sense, the use of human behaviour and actions in non-living agents, as robots, can perform a significant influence on person responses. Therefore, the use of persuasion strategies that use personality at its core should be thoughtfully, peacefully and humanistically designed, not to be used to spread fake messages.

\section{ACKNOWLEDGMENTS}

We would like to thank the State University of Rio Grande do Norte (UERN) and National Council for Scientific and Technological Development (CNPq) program Science without Border: 201833/2014-0 - Brazil, for PhD grants to the first author. This work was also supported by Fundação para a Ciência e a Tecnologia: (FCT) UIDB/50021/2020. 


\section{REFERENCES}

[1] A. Aly and A. Tapus. 2013. A model for synthesizing a combined verbal and nonverbal behavior based on personality traits in human-robot interaction. In Proceedings of the 8th ACM/IEEE international conference on Human-robot interaction. IEEE Press, 325-332.

[2] J.K. Burgoon, L.K. Guerrero, and V. Manusov. 2011. Nonverbal signals. The SAGE handbook of interpersonal communication (2011), 239-280.

[3] A. Pacheco C and C. Martinho. 2019. Alignment of Player and Non-Player Character Assertiveness Levels. In Proceedings of the AAAI Conference on Artificial Intelligence and Interactive Digital Entertainment, Vol. 15. 181-187.

[4] C.S. Carver and M.F. Scheier. 2012. Perspectives on personality. Pearson Education.

[5] A. Cerekovic, O. Aran, and D. Gatica-Perez. 2014. How do you like your virtual agent?: Human-agent interaction experience through nonverbal features and personality traits. In International Workshop on Human Behavior Understanding. Springer, $1-15$

[6] V. Chidambaram, Y. Chiang, and B. Mutlu. 2012. Designing persuasive robots: how robots might persuade people using vocal and nonverbal cues. In Proceedings of the seventh annual ACM/IEEE international conference on Human-Robot Interaction. ACM, 293-300.

[7] P.T. Costa and R.R. McCrae. 1992. Revised NEO personality inventory (NEO PI$R$ ) and NEP five-factor inventory (NEO-FFI): professional manual. Psychological Assessment Resources Lutz, FL.

[8] C. Kidd D and C. Breazeal. 2008. Robots at home: Understanding long-term human-robot interaction. In Intelligent Robots and Systems, 2008. IROS 2008 IEEE/RSf International Conference on. IEEE, 3230-3235.

[9] J.P. Dillard and L. Shen. 2013. The Sage handbook of persuasion. Sage.

[10] T.R. Elliott and S.E. Gramling. 1990. Personal assertiveness and the effects of social support among college students. Journal of counseling psychology 37,4 (1990), 427

[11] R.H. Gass and J.S. Seiter. 2015. Persuasion: Social influence and compliance gaining Routledge.

[12] A. Gelfert. 2018. Fake news: A definition. Informal Logic 38, 1 (2018), 84-117.

[13] A.S. Ghazali, J. Ham, E. Barakova, and P. Markopoulos. 2018. The influence of social cues in persuasive social robots on psychological reactance and compliance. Computers in Human Behavior 87 (2018), 58-65.

[14] G.H. Gudjonsson. 1988. Interrogative suggestibility: Its relationship with assertiveness, social-evaluative anxiety, state anxiety and method of coping. British Journal of Clinical Psychology 27, 2 (1988), 159-166.

[15] J. Ham, R.H. Cuijpers, and J. Cabibihan. 2015. Combining robotic persuasive strategies: the persuasive power of a storytelling robot that uses gazing and gestures. International fournal of Social Robotics 7, 4 (2015), 479-487.

[16] O. John, E. Donahue, and R. Kentle. 1991. The big five inventory: versions 4a and 54.

[17] S.M. Kassin. 2003. Essentials of psychology. Prentice Hall.

[18] C.E. Kimble and S.D. Seidel. 1991. Vocal signs of confidence. Fournal of Nonverbal Behavior 15, 2 (1991), 99-105. https://doi.org/10.1007/BF00998265

[19] J. Kkedzierski, R. Muszyński, C. Zoll, A. Oleksy, and M. Frontkiewicz. 2013. EMYS-emotive head of a social robot. International fournal of Social Robotics 5 , 2 (2013), 237-249.

[20] J. Kory. 2014. Storytelling with robots: Effects of robot language level on children's language learning. Ph.D. Dissertation. Massachusetts Institute of Technology.

[21] J. Kory and C. Breazeal. 2014. Storytelling with robots: Learning companions for preschool children's language development. In Robot and Human Interactive Communication, 2014 RO-MAN: The 23rd IEEE International Symposium on. IEEE, 643-648.
[22] K.M. Lee, W. Peng, S. Jin, and C. Yan. 2006. Can robots manifest personality?: An empirical test of personality recognition, social responses, and social presence in human-robot interaction. Fournal of communication 56, 4 (2006), 754-772.

[23] M.K. Lee, J. Forlizzi, S. Kiesler, P. Rybski, J. Antanitis, and S. Savetsila. 2012. Personalization in HRI: A longitudinal field experiment. In Human-Robot Interaction (HRI), 2012 7th ACM/IEEE International Conference on. IEEE, 319-326.

[24] J. Li. 2015. The benefit of being physically present: A survey of experimental works comparing copresent robots, telepresent robots and virtual agents. International fournal of Human-Computer Studies 77 (2015), 23-37.

[25] S. C. Matz, M. Kosinski, G. Nave, and D. J. Stillwell. 2017. Psychological targeting as an effective approach to digital mass persuasion. Proceedings of the National Academy of Sciences 114, 48 (2017), 12714-12719. https://doi.org/10.1073/pnas. 1710966114 arXiv:https://www.pnas.org/content/114/48/12714.full.pdf

[26] A. Muhammad, M. Hamid, and N. Muhammad. 2012. Persuading students for behavior change by determining their personality type. In 2012 15th International Multitopic Conference (INMIC). IEEE, 439-449.

[27] I.B. Myers and P.B. Myers. 1980. Gifts Differing: Understanding Personality Type. Davies-Black Pub. https://books.google.pt/books?id=D3V-AAAAMAAJ

[28] R. Orji, R.L. Mandryk, and J. Vassileva. 2015. Gender, age, and responsiveness to Cialdini's persuasion strategies. In International Conference on Persuasive Technology. Springer, 147-159.

[29] R. Paradeda, M.J. Ferreira, C. Martinho, and A. Paiva. 2017. Using Interactive Storytelling to Identify Personality Traits. In International Conference on Interactive Digital Storytelling. Springer, 181-192.

[30] R. Paradeda, M.J. Ferreira, C. Martinho, and A. Paiva. 2018. Communicating Assertiveness in Robotic Storytellers. In Interactive Storytelling, Rebecca Rouse, Hartmut Koenitz, and Mads Haahr (Eds.). Springer International Publishing, Cham, 442-452.

[31] R. Paradeda, M.J. Ferreira, C. Martinho, and A. Paiva. 2018. Would You Follow the Suggestions of a Storyteller Robot?. In International Conference on Interactive Digital Storytelling. Springer, 489-493.

[32] R. Paradeda, M.J. Ferreira, R. Oliveira, C. Martinho, and A. Paiva. 2019. The Role of Assertiveness in a Storytelling Game with Persuasive Robotic Non-Player Characters. In Proceedings of the Annual Symposium on Computer-Human Interaction in Play. October 22-25, 2019. Barcelona, Spain.

[33] R. Paradeda, M.J. Ferreira, R. Oliveira, C. Martinho, and A. Paiva. 2019. What Makes a Good Robotic Advisor? The Role of Assertiveness in Human-Robot Interaction. In Proceedings of the the 11th International Conference on Social Robotics. October 26-29, 2019. Madrid, Spain.

[34] R. Paradeda, M. Hashemian, R. Rodrigues, and A. Paiva. 2016. How Facial Expressions and Small Talk May Influence Trust in a Robot. Springer International Publishing, Cham, 169-178. https://doi.org/10.1007/978-3-319-47437-3_17

[35] R. Paradeda, C. Martinho, and A. Paiva. 2017. Persuasion Based on Personality Traits: Using a Social Robot As Storyteller. In Proceedings of the Companion of the 2017 ACM/IEEE International Conference on Human-Robot Interaction (Vienna, Austria) (HRI '17). ACM, New York, NY, USA, 367-368. https://doi.org/10.1145/ 3029798.3034824

[36] R. Picard. 1995. Affective computing. Perceptual Computing Section, Media Laboratory, Massachusetts Institute of Technology (1995).

[37] A. Powers, S. Kiesler, S. Fussell, and C. Torrey. 2007. Comparing a computer agent with a humanoid robot. In Human-Robot Interaction (HRI), 2007 2nd ACM/IEEE International Conference on. IEEE, 145-152.

[38] K. Ryokai, C. Vaucelle, and J. Cassell. 2003. Virtual peers as partners in storytelling and literacy learning. Fournal of computer assisted learning 19, 2 (2003), 195-208.

[39] A. Tapus and M. Matarić. 2008. User personality matching with a hands-off robot for post-stroke rehabilitation therapy. In Experimental robotics. Springer, 165-175. 